Manuelle Medizin 2014 · 52:95-96

DOI 10.1007/s00337-014-1104-6

Online publiziert: 4. April 2014

c) Springer-Verlag Berlin Heidelberg 2014
L. Beyer ${ }^{1} \cdot$ E.J. Seidel ${ }^{2}$

1 Ärztehaus Mitte, Jena

2 Ambulantes Rehabilitations- und Rückenzentrum, Sportmedizinisches

Untersuchungszentrum DOSB, Sophien- und Hufeland-Klinikum Weimar

\title{
Hohes Potenzial positiver Einflüsse bei der Sportlerbetreuung
}

Liebe Leserin, lieber Leser,

manualmedizinische Verfahren werden in der Sportlerbetreuung von den Anwendern mehrheitlich als positiv angesehen.

In der Sportmedizin stehen neben den therapeutischen Interventionsmitteln der manuellen Medizin die differenzialdiagnostischen Aspekte der manuellen Medizin am Bewegungssystem im Vordergrund. Die Einschätzung manualmedizinischen Könnens reicht vom positiven Einfluss bei der Rehabilitation von Sportverletzungen über die Prophylaxe von Sportverletzungen bis hin zur Möglichkeit der Leistungssteigerung. Grundlage sind vorwiegend neurophysiologische Erklärungsmodelle, die nahelegen, dass funktionelle Störungen zu einer veränderten Gelenkbeweglichkeit und muskulären Koordination führen, die durch geänderte Bewegungsabläufe ein höheres Verletzungsrisiko und eine verminderte Leistungsfähigkeit zur Folge haben.

Als definitiv nachgewiesen kann der positive Einfluss der Manualmedizin in der Behandlung von Sportverletzungen angesehen werden. Klett zeigt in seinem Beitrag Möglichkeiten und Grenzen auf.

Bei der Betreuung von Sportlern in den verschiedenen Disziplinen werden vom behandelnden Arzt und Physiotherapeuten neben den Kenntnissen der manuellen Diagnostik, der funktionellen Anatomie sowie der individuellen Gegebenheiten der Anthropometrie auch Kenntnisse zu den Anforderungen und Besonderhei- ten der Sportarten gefordert. Zu Letzteren gehören die typischen Bewegungsabläufe, das erforderliche sportartspezifische Training und seine individuellen Gestaltungsmöglichkeiten.

Der Anatom und Sportmediziner $K$. Tittel aus Leipzig beschäftigt sich seit 1952 intensiv mit den Fragen funktionellanatomischer und sportmedizinischer Aspekte der muskulären Verkettungen und Funktionsprinzipien der Bewegung, insbesondere auch unter den Besonderheiten der sportlichen Belastung. Heute reicht es nicht mehr aus, Antagonisten und Agonisten zu kennen, deren Kraft bzw. Dehnfähigkeiten zu testen und daraus resultierend das Therapieregime auf Verkürzungen und Abschwächungen zu konzentrieren. Es muss genauer hingeschaut werden, wenn es darum geht, zu erkennen, durch welches sportliche Training muskuläre und arthromuskuläre Dysbalancen verursacht werden. Erst dann wird es möglich, ihre Bedeutung für die Vermeidung von Verletzungen bzw. für die besondere Disposition für Verletzungen der Sportler $\mathrm{zu}$ verstehen. $\mathrm{Zu}$ analysieren ist das koordinative Zusammenspiel von tonisch und phasisch arbeitenden Muskeln als konkret wirksam werdende Antagonisten und Agonisten. So sind beispielsweise Kokontraktionen am Kniegelenk bei der Bewegungsausführung dazu geeignet, Bandstrukturen muskulär zu schützen. Sie sind durch reine Kraft- und Dehnungsübungen nicht zu erzielen und können nur durch ein koordinatives, propriozeptives
Training dieser dem Gelenkschutz dienenden Kokontraktoren erarbeitet werden. Der Beitrag von Tittel zeigt uns die Bedeutung dieses Zusammenspiels.

\section{) Zu beachten sind Individualität und sportartspezifische Normen}

Der Beitrag zeigt aber auch, dass im Sport nicht das allgemeine Normprinzip bei der Anwendung der manuellen Medizin umgesetzt werden darf, sondern dass eine auf explizite Kenntnis sportmotorischer Bewegungsabläufe basierende sportartspezifische Norm vorauszusetzen ist. Eine Mitgestaltung des Trainings durch den Manualtherapeuten/Arzt ist erforderlich. Ein paralleles Beispiel hierfür sind muskuläre und arthromuskuläre Dysbalancen von Musikern, bei denen ebenfalls die Normprinzipien aus Alltagsfunktionen nicht auf den Patienten bzw. betreuten Musiker übertragen werden können. Wer sich als Behandler mit den leistungsphysiologischen Prinzipien in der Sportmedizin, insbesondere der biomechanischen Funktionsdiagnostik bei bestimmten Sportarten, nicht auskennt, kann durch falsche Normvorstellungen einem zu betreuenden Sportler auch schaden bzw. zu dessen Leistungsminderung beitragen.

Weitere Beiträge beleuchten manualmedizinische und sportmedizinische Aspekte in verschiedenen Sportarten. Hüsers 
et al. beschäftigen sich mit der Frage „Sind langwierige Sprunggelenksverletzungen mit Beckendysfunktionen assoziiert?", Seidel u. Tittel beschreiben Kreuzbandverletzungen im Frauensport und Dehoust schildert ein typisches Fallbeispiel aus dem Triathlonsport.

Lorch et al. stellen fest, dass die Ruptur des Lig. teres aufgrund vieler Gemeinsamkeiten mit dem vorderen Kreuzband des Kniegelenks eine zunehmend anerkannte Ursache für dauerhafte Schmerzen in der Hüfte nach Verletzung oder Degeneration darstellt, die sich bei Sportlern als dritthäufigste Verletzung äußert.

Eine ausführliche Übersicht zum vorderen Knieschmerz gibt Biesenbach. In sportmedizinischen Schwerpunktpraxen gehören Symptome in der vorderen Knieregion zu den am häufigsten genannten Beschwerden. Rezidivierende Beschwerden können zu langen Unterbrechungen sportlicher Aktivität führen. Der Autor stellt die in der Literatur aufzufindenden diagnostischen Algorithmen und therapeutischen Konzepte vor.

Die Beiträge sollen dem betreuenden Manualmediziner und -therapeuten wertvolle Hinweise zu sportartspezifisch auftretenden muskulären und arthromuskulären Dysbalancen geben.

Außerhalb des Themas „Manuelle Medizin und Sport" möchten wir Sie auf die von der FIMM erarbeiteten „Leitlinien zur Grundausbildung und Sicherheit in manueller/muskuloskeletaler Medizin“" aufmerksam machen, deren erster Teil in diesem Heft abgedruckt ist.

Ihre

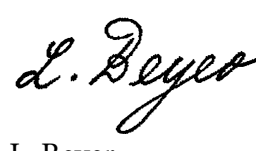

L. Beyer

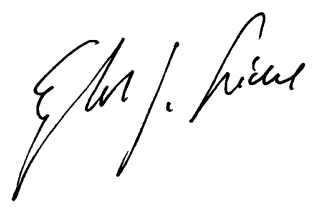

E. Seidel

\section{Korrespondenzadresse}

Prof. Dr. L. Beyer

Ärztehaus Mitte

Westbahnhofstr. 2,

07745 Jena

lobeyer@t-online.de

Prof. Dr. E.J. Seidel

Ambulantes Rehabilitations-

und Rückenzentrum,

Sportmedizinisches Untersuchungszentrum

DOSB, Sophien-

und Hufeland-Klinikum Weimar

Henry-van-de-Velde-Str. 2,

99425 Weimar

Interessenkonflikt. L. Beyer und E. Seidel geben an, dass kein Interessenkonflikt besteht.

\section{KBV-Bericht Ärzte sind überzeugte QM-Täter}

Gutes Zeugnis für Vertragsärzte: Das QM läuft in mehr als drei Vierteln der Praxen rund. Aber auch in Sachen DMP und Qualitätszirkel legen Ärzte nach.

Ob bei der Fortbildungs- oder QM-Pflicht: Nachzügler in Sachen Qualitätsanforderungen und QM-Umsetzung sind im ambulanten Bereich nur noch eine seltene Gattung. Über zwei Drittel der Vertragsärzte befanden sich 2012 bei ihrem praxisinternen Qualitätsmanagement (QM) im Soll. Acht Prozent hatten ihr Soll sogar übererfüllt. Gerade einmal 23 Prozent der Ärzte hinkten den Vorgaben für ihr QM noch hinterher. So das Ergebnis des aktuellen Qualitätsberichts der KBV.

Ein Blick in die KBV-Statistik zum Stand des QM in den Vertragsarztpraxen verrät aber noch mehr: In nur 17 Praxen wurde 2012 eine Pflichtberatung in Sachen QM vorgenommen. Fast 68 Prozent der Praxen hatten 2012 mit der Weiterentwicklung ihres QM begonnen. Knapp über ein Prozent hatten sich noch gar nicht mit dem Qualitätsmanagement beschäftigt.

Noch ein bisschen besser sehen die Zahlen bei der ärztlichen Fortbildungspflicht aus. Diese gilt seit Juli 2004, damit endete die erste Fünf-Jahres-Nachweispflicht am 30. Juni 2009. Damals erfüllten prompt 94,3 Prozent der Ärzte ihre Fortbildungspflicht und konnten die geforderten 250 Fortbildungspunkte nachweisen. Zusammen mit der zweijährigen Nachreichfrist erreichten sogar über 99 Prozent der Ärzte die 250 Punkte. 2012 stieg die Zahl derer, die ohne Nachreichfrist ihre Punkte erzielten auf 96,5 Prozent.

Dass sich Ärzte zunehmend für mehr Qualität in der Versorgung einsetzen, zeigt aber auch die Entwicklung der ärztlichen Qualitätszirkel: Mehr als 9500 zertifizierte Qualitätszirkel, neun Prozent mehr als 2011, zählte die KBV für das Jahr 2012. Insgesamt 68.000 Ärzte nahmen an den Zirkeln teil.

Quelle:

ÄrzteZeitung, www.aerztezeitung.de 